\title{
A Novel Feature Extraction for Complementing Authentication in Hand-based Biometric
}

\author{
Mahalakshmi B S \\ Assistant Professor \\ Department of Information Science and Engineering \\ BMS College of Engineering, Bangalore, India
}

\author{
Sheela S V ${ }^{2}$ \\ Professor \\ Department of Information Science and Engineering \\ BMS College of Engineering, Bangalore, India
}

\begin{abstract}
With an increasing usage of hand-based biometrics in authentication system, there is a need to evolve up with more potential security owing to increasing evolution of threats. The security of the hand authentication system completely depends upon uniqueness and distinct selection of features from hand image which has the properties of robustness, fault tolerance, and simpler implication. Review of existing feature extraction literatures shows more inclination towards sophisticated process as well as it also suffers from various other limitation. Therefore, this manuscript resolves this limitation by presenting a novel model of feature extraction which is carried out in more progressive form and less iterative form, unlike existing approaches. The proposed system achieves its research goal by introducing simplified feature extraction operation via storage, blurring, color space conversion, binary image conversion, the modelling aspect of the study emphasizes on image enhancement along with fuzzification for yielding more efficient result. An experimental study has been carried out using Python considering hand-biometric dataset in order to carry analysis where the outcome shows significant supportability over any palmprint recognition system. The study outcome is compared with most standard implementation of feature extraction to find that proposed system offer better accuracy performance in contrast with existing system.
\end{abstract}

Keywords-Biometric; security; feature extraction; hand geometric; authentication; palmprint recognition

\section{INTRODUCTION}

Authentication is defined as the process of verifying something to be genuine. In our case however, we are trying to verify if the identity of the person is genuine or not. Biometric authentication is a method of authentication where the system focuses on 'who you are' rather than 'what you know' (password) [1]. Biometrics is defined as the biological measurements which will identify an individual [2]. With these studies we can conclude that more unique the data is, more robust the system will be in identifying the individuality. Thus it can be concluded that the system which efficiently identify a person with the data of 'who he is' (bio-metric) rather than what he knows (password) is more secure as passwords are considered to be more insecure compared to biometrics [3]. Even if there are many types of modalities that can be collected for biometric authentication of a person, obtaining the hand geometry require less complex systems of input data acquisition in comparison with other modalities [4]. In the complete mechanism of authentication mechanism, feature extraction plays a vital role that contributes towards dimensional reduction as well as it also offers significant information for precisely carry out the next step of authentication operation. The generalized definition of feature could be stated as a functional information of one or more set of information that when subjected to computation yields at quantifying potential characteristic of an object. Theoretically, a particular form of an image feature can be defined with respect to particular structure within an image that is always feasible to represents in multiple mechanisms. It is to be noted that selection of feature representation can be potential concern while developing a computer vision system. There could be dependencies towards adopting higher level of information in the structure of a feature in order to find a solution to the problem; however, it will require more processing attempts. A complete image or a segment of an image can be represented by the feature vector. This can be accomplished by exploring measurement of the group of features. Usually, a feature vector can be n-dimensional vector which consist of such measurements. Some of the widely used conventional features are Zernike Moments [5], Local orientation histograms [6], local brightness [7], binary object feature [8], etc. However, this will eventually differ from one to another image. From the palmprint image viewpoint, there are various forms of features e.g. wrinkles, textures, ridges, valleys, minute points, pores, ridge width, etc. Conventional approaches [9], [10] mainly make use of line-based methods as elementary representation of palmprint. Studies claims to offer stabilized authentication of palm using principle lines. It should be also noted that a high resolution image of palm print is required in order to perform a detection of features from palm print. The challenges increases more as palm print images are basically of two types viz. two and three dimensional palm images. Two dimensional palm images are further classified into low and high resolution image where low-resolution two dimensional palm print images are further classified into contact-based and contactless forms. With an increasing usage of hand-based biometric system in commercial products and services, it is essential to ensure that a robust modelling be carried out in this regards. Apart from this, with the presence of archives of literatures towards biometric authentication, not much emphasize is offered towards feature extraction. Hence, this loophole in existing system motivates to carry out a novel research work to address the above mentioned issues of existing system.

This paper introduces a novel and simplified mechanism of feature extraction of hand where the emphasis is offered 
towards achieving cost effective implementation process unlike existing sophisticated extraction process. The paper presents a simplified model which emphasize on the feature extraction process in order to assist a robust form of biometric-based authentication system. Existing approaches towards biometric authentication system mainly emphasize on using complex form of feature extraction process which could not effectively balance between computational efficiency and accuracy at same time. At the same time, it is also noticed that not much emphasis is offered towards the contextual informative contents with respect to foreground and background. Noninclusion of this fact will eventually lead to outliers in the authentication system. However, it should be noted that proposed system doesn't develop any form of authentication system; it only introduces a feature extraction mechanism which is meant to be used for any form of hand-based biometric system in future. Hence, this leads to evolution of certain research questions:

- What are the dominant level of features essential for hand-based biometric system to ascertain both accuracy and computational efficiency?

- How to find the artifacts and mitigate them for the fluctuation of illumination state in foreground and background to ensure better removal of outliers?

Without addressing the above two critical research question, developing a feature extraction method will not encapsulate the practical issues pertaining to the authentication system in biometric. The proposed system also emphasize on image enhancement process which offers a superior form of accuracy in contrast to existing scheme of recognition system of hand. The organization of this manuscript is as follows: Section II discusses about the existing approaches of feature extraction followed by discussion of research problem that are confirmed after reviewing existing approaches in Section III. Further, proposed system design is discussed in Section IV followed by discussion of result analysis in Section $\mathrm{V}$ and conclusion in Section VI.

\section{RELATED STUDIES}

This section discusses about all the significant research work carried out in recent time towards feature extraction process. It should be known that feature extraction is an intermediate process within recognition of the palm. Hence, this part of the studies discusses about majority of studies that has considered hand recognition system based on palm geometry. While equal emphasis is also given for other image object-based recognition system in order to understand the effectiveness of unique feature extraction approaches.

Existing literatures has consideration of multispectral palm images for recognition process as seen in work of Attallah et al. [11]. The study has emphasized on using spiral feature as well as Linear Binary Pattern for the purpose of feature extraction. Finally, K-nearest neighborhood is used for matching purpose. The outcome is analyzed with multiple dataset to prove its effectiveness. However, the owing to statistical computational adoption, the study doesn't emphasize on possibilities of outliers when color images are taken. Most recent, a unique feature extraction process is discussed by Bakheet and AlHamadi [12] where both region and boundary based process of feature extraction is discussed in order to construct a multimodal descriptor from hand silhouette. Further supervised learning approach is used for training this feature in order to carry of classification. The works carried out by Deshpande et al. [13] have used Harris Corner Detection and Discrete Wavelet Transform in order to carry out feature extract from palm print images. The formulated feature is transformed in binary matric that is subjected for comparison while performing training.

The work of Zhang et al. [14] have used an iterative learning scheme for extracting hidden information in the form of feature.

The work of Yang et al. [15] discusses about the importance of offset feature while performing analysis of feature. The study has used infinite Dirichlet process in order to carry out analysis.

Existing system of recognition also addressed the problems associated with partial occlusion. The study of Liu et al. [16] has used a graph matching mechanism for addressing this problem of recognition system of dorsal hand. The authors have used conventional shape-based feature extraction mechanism for recognition of vein along with edge attributes. Existing literatures has offered a solution towards adaptability problems in palmprint recognition. The work of Zhao et al. [17] has used Least Square Regression as a mechanism for feature extraction. The work carried out by Gupta and Gupta [18] has presented a study of authentication model using multibiometrics of hand images in the form of hand geometry, dorsal vein of palm, and slap fingerprints. The essential feature in this case are location of fingers which is carried out using segmentation-based process. Nearly similar form of study is also discussed by Izakian et al. [19] where segmentation using trajectories are adopted for carrying out feature extraction. The feature in this study is the alterations of the specific movement profile captured in sliding window. Existing studies has been also reported to make use of Doppler map for carrying out feature extraction. The work carried out by Ryu et al. [20] have used evolutionary approach in order to carry out feature analysis. The study has considered feature specific to radar characteristic along with statistical outcomes to be used in machine learning approach. The feature extraction in this study is carried out using low level descriptor, tracking scattering center, and obtaining connectivity between channels.

Most recently, there are studies which are carried out using machine learning approach towards processing features from hand images. The study carried out by Xin and Wang [21] has discussed about utilization of $\mathrm{CNN}$ for assisting in classification of image considering different requirements of feature extraction. Such study is carried out by Du et al. [22] where Convolution Neural Network (CNN) is used for recognition of hand gesture. This study make use of microDoppler feature from target areas in order to generate more logical and adaptive feature. CNN was used further to improve upon the recognition system. Similar category of usage of CNN is also seen in work of Abd-Ellah et al. [23] where CNN is used for high-level feature extraction in order to obtain 
feature map. Exactly similar approach is also reported in work of $\mathrm{Li}$ et al. [24] have carried out the study towards feature extraction using Micro-Doppler associated with recognition of hand gesture. The study has also used orthogonal-based matching map for performing this extraction process where further CNN is used for training.

Adoption of CNN is also reported in work of Ghrabat et al. [25] integrated with search optimization scheme of evolutionary approach. The feature in this study are the color and texture. This is accomplished using co-occurrence matrix of gray level and image intensity for extracting color and texture feature respectively. Further K-means clustering is used for grouping the feature while classification is carried out by Support Vector Machine (SVM) and CNN. Existing mechanism has also witnessed usage of $\mathrm{CNN}$ for feature extraction associated with semantic aspect of recognizing vein. This work is reported by Pan et al. [26] where all the feature map are concatenated where the selection of optimal feature is carried out on the basis of semantic weights. A unique adoption of machine learning approach was discussed by Wu et al. [27] towards optimizing the extracted feature. The scheme make use of inferential statistics and neural network in order to carry out recognition. The features used in this study are time-frequency domain, frequency domain, and time domain feature. Yoo et al. [28] have carried out used recurrent neural network in order to study articulation of hand by extracting sequential feature of joints in fingers. Further Long Short Term Memory (LSTM) was reported to be used in study of Yuan et al. [29] for deep feature extraction. The work carried out by Wibisono and Mursanto [30] have also presented a sophisticated feature extraction scheme using deep learning.

Hence, there exists various studies towards feature extraction in existing system. The next section discusses about research problems associated with it.

\section{RESEARCH PROBLEM}

After reviewing the existing literatures towards feature extraction considering hand geometry, various conclusive remarks were obtained associated with its downsides. Following were the research problems that has been identified and addressed in current work:

- Complex Mechanism of Feature Extraction: Existing system are mainly reported to use spiral feature as well as Linear Binary Pattern, Harris Corner Detection and Discrete Wavelet Transform, infinite Dirichlet process, segmentation using trajectories, micro-Doppler feature, etc. All these approaches are quite conventional and sophisticated in its operation with respect to various traits of hand geometry. Owing to inclusion of maximum number of operational steps to carry out feature extraction, these methods may offer poor scalability in its performance.

- More usage of Learning Operation: Machine learning and its different variants were discussed for improving the performance of feature extraction. However, machine learning methods implemented in existing schemes are more focused on achieving accuracy and less on offering granularity in feature extraction from dynamic environment. Moreover dependencies of trained data will further act as an impediment towards feature extraction if images with multiple orientation and modalities are used.

- Biased Adoption of Illumination Factor: Majority of the existing approaches considers the illumination factors over the foreground object and not the background object. The silhouette generated by the hand structure also induce variation in background illumination factor and significant results in outliers (conjoining fingers). Therefore, there is a need of using a simplified scheme which offers equal importance to the illumination factor for extracting precise feature.

- Less Preference to Image Enhancement: Majority of the existing implementation follows a dual steps of operation viz. feature extraction after preprocessing and subjecting the feature for further process of authentication or recognition. However, there are few literatures to highlight further importance for enhancing the image, which is also a challenging step to be carried out. Without this operation, it is a challenging process in order to ensure better visual quality of the outcome image. It also affects the accuracy of the outcome image.

- Common preprocessing steps: Not much literatures has witnessed the use of unique and simplified preprocessing operation. There is a need to preprocessing to eliminate noise in simplified manner, there is a need to consider different form of color space apart from conventional RGB, and also, there is a need to evolve up with a different binarization scheme. At present, there are very studies that has emphasized on such operations while performing feature extraction.

It should be noted that research problem in hand is associated with the feature extraction. All the above mentioned points represents the limiting factors of existing approaches. Out of all this, the first critical problem which requires to be prioritized primarily is associated with preprocessing operation. A better and simplified preprocessing operation will eventually lead to better artifact removal from the biometric input given, which unfortunately lacks in existing approach. This will also contribute towards addressing enhancement issues in an image. The second essential problem is towards dealing with the illumination factor without which proposed model will not be able to deal with different variants of images of hand-based images. The next section discusses about the system design implemented in proposed system as solution.

\section{SYSTEM DESIGN}

The proposed study is focused on feature extraction methodology rather than the recognition model itself. The hypothesis is that if the feature extraction is carried out in efficient manner than it will positively affect the recognition modelling too. The system design of the proposed system is motivated from the work carried out by Afifi et al [31]. A Convolution Neural Network (CNN) system is used to extract 
the features and Support Vector Machine (SVM) is used to authenticate the person in existing system. When a CNN is being trained, its performance can be enhanced if right preprocessing steps are added to it [32]. It should also be noted that feature enhancement will improve the performance of the recognition system without making any fundamental changes to the system itself [33]. Though the explicit method for handling the contrast and enhancement can be used if there exists a varying lighting condition, which is the sole purpose of the proposed system. In the proposed model, a novel type of preprocessing system is being proposed which will enhance the performance of the hand geometry recognition and authentication algorithm. As shown in Fig. 2, the proposed system contains five distinct modules viz. i) image blurring, ii) HSV conversion iii) Fuzzy logic binary converter, iv) contour detector v) image enhancer. The purpose of all above modules is to detect the important features in the hand geometry and enhance them so that they are better exposed to next algorithm which will be used for classification/recognition. The block diagram of the system is as shown in the Fig. 1.

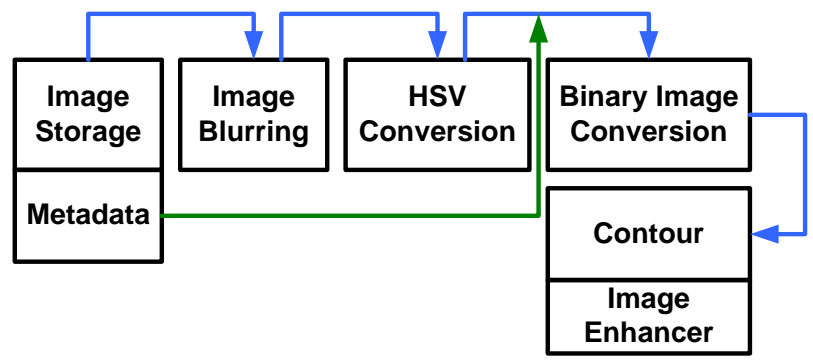

Fig. 1. Proposed Preprocessing System.

The brief outlines of above exhibited blocks of operation are as follows:

\section{A. Image Storage}

This block of operation retains images associated with palm geometries that is considered for study implementation. The proposed system uses $11 \mathrm{~K}$ hand dataset that consists of 11,000 images of hands belonging to 190 people. This also provides additional details about the images in the form of metadata [31]. The metadata are used further prior to the process of binary image conversion. Since in $11 \mathrm{~K}$ hands dataset, even the metadata is given, It helps in present study to recognize if a person is having any accessory while processing the image. If person is wearing a ring or nail polish, the results may vary hence these taken into account during fuzzy image binarization.

\section{B. Image Blurring}

The proposed system implements image blurring in order to eliminate certain features as well as to eliminate noise [34]. Amongst various techniques used for blurring, the proposed system implements Gaussian blurring approach. It is the result of blurring the image with the help of Gaussian function. The one-dimensional Gaussian function is mathematically expressed as follow:

$G(x)=\frac{1}{\sqrt{2 \pi \sigma^{2}}} e^{-\frac{x^{2}}{2 \sigma^{2}}}$

However, while dealing with images, the formula must be expanded to two dimensions. The two-dimensional mathematical expression of proposed blurring is now as follows:

$G(x, y)=\frac{1}{\sqrt{2 \pi \sigma^{2}}} e^{-\frac{x^{2}+y^{2}}{2 \sigma^{2}}}$

In the above expression (2), the variable $\sigma$ represents the window size. In proposed study, window size of 3 is selected and the above mathematical expression is applied to every pixel of the image. Due to Gaussian blur, noises in the image are removed and also reflection caused due to shininess of aged skin and blood veins are also removed [35].

\section{HSV Conversion}

This is the third step of operation in proposed system where color space of Hue, Saturation and Value (HSV) is deployed. The prime justification of using HSV is because it is a standard to represent a color in a more human understandable way. In proposed study, the image is converted into HSV format so that various colors of skin can easily be represented in HSV format rather than regular RGB format. To detect human skin, HSV is more advantageous compared to RGB since it is easy to calculate and ignore ambient light [36]. In order to convert RGB to HSV, the channels must be flipped if the implementation is being done in OpenCV as it used BGR format instead of RGB. In order to do this, all three colors must be normalized using minmax scaler. Formula for same is shown below.

$Y^{\prime}=\frac{Y-Y_{\min }}{Y_{\max }-Y_{\min }}$

However it must also be noted that pixel values always range between 0 to 255 hence $Y_{\max }$ is always retained at the value of 255 and $Y_{\min }$ value is retained at 0 . This will yield to in terms of following mathematical expression.

$R^{\prime}=\frac{R}{255}, G^{\prime}=\frac{G}{255}, B^{\prime}=\frac{B}{255}$

Once all three color values are converted, the following algorithm is used to make the conversion. In the algorithm which is being used, the HSV conversion is done with the help of modulus function hence the conversion happens in a much faster way as contrary to using trigonometric functions. First, the algorithm calculates Hue by calculating the angle of color from nearest elementary color (RGB) Saturation is calculated as a measure of percentage difference between most dominant and least dominant color and finally value is nothing but the value of dominant color. 


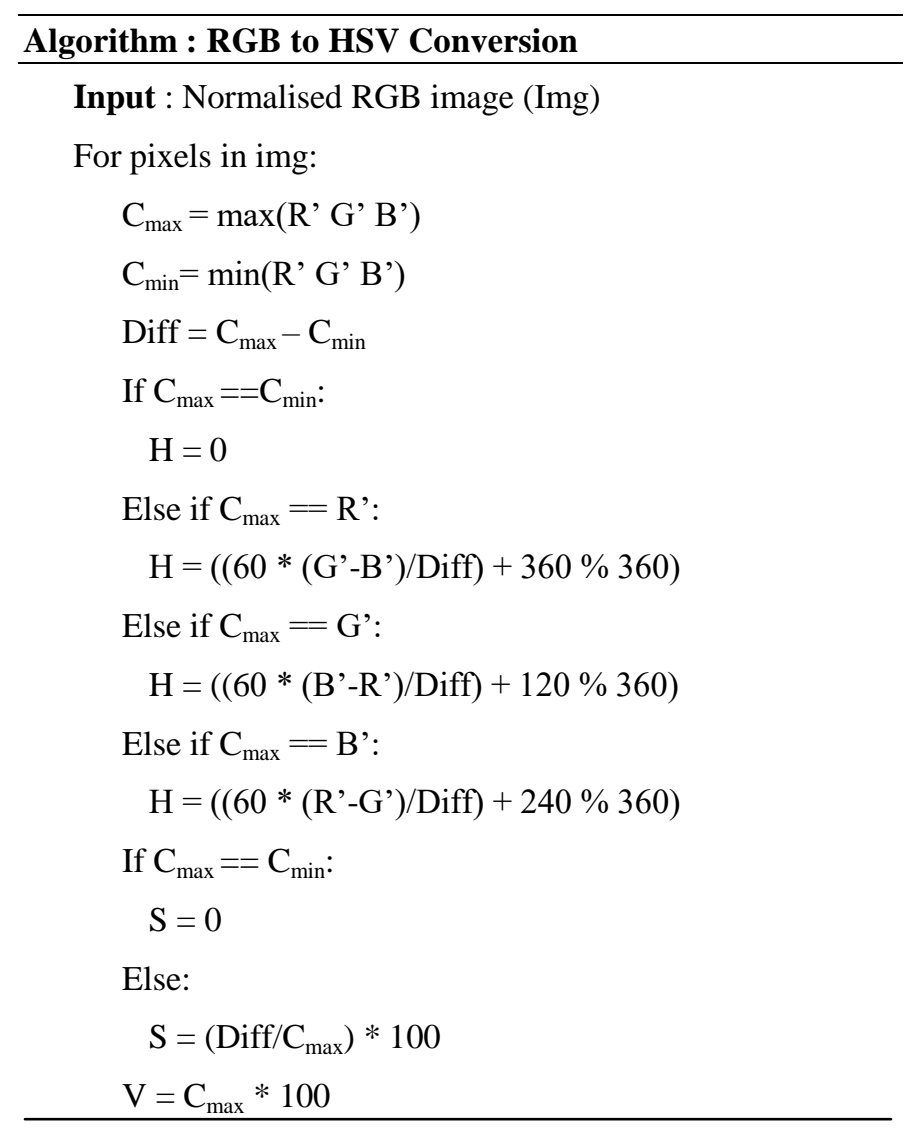

\section{Image Binarization}

The image must be converted to binary image in order to make it easy for processing. In case of this study, the HSV values are converted to binary values. The complete process of image binarization is shown in Fig. 3.

According to Fig. 2, the proposed system takes an input image and considers all the pixels present in an image for further processing. The next step of operation is to check if the HSV value is more than upper value of the threshold $\mathrm{T}_{\text {up }}$. If this condition is satisfied, the proposed system checks of the value of HSV is more than lower threshold $\mathrm{T}_{\text {low. }}$. In either cases of the condition is not satisfied than proposed system carry out fuzzification followed by a conditional check if the adjacent pixel $\mathrm{P}_{\mathrm{ad}}$ is white. If the adjacent pixel is found to be white than the proposed model converts them in black or else it makes it white. In the exhibited Fig. 3, the process of fuzzification is the process where probability of the pixel being skin or not skin. In order to do this an algorithm will run in the background and decide the values of Hue so that the algorithm works for various skin tones. The proposed system performs the following steps of implementation towards hand image for eigenvectors.

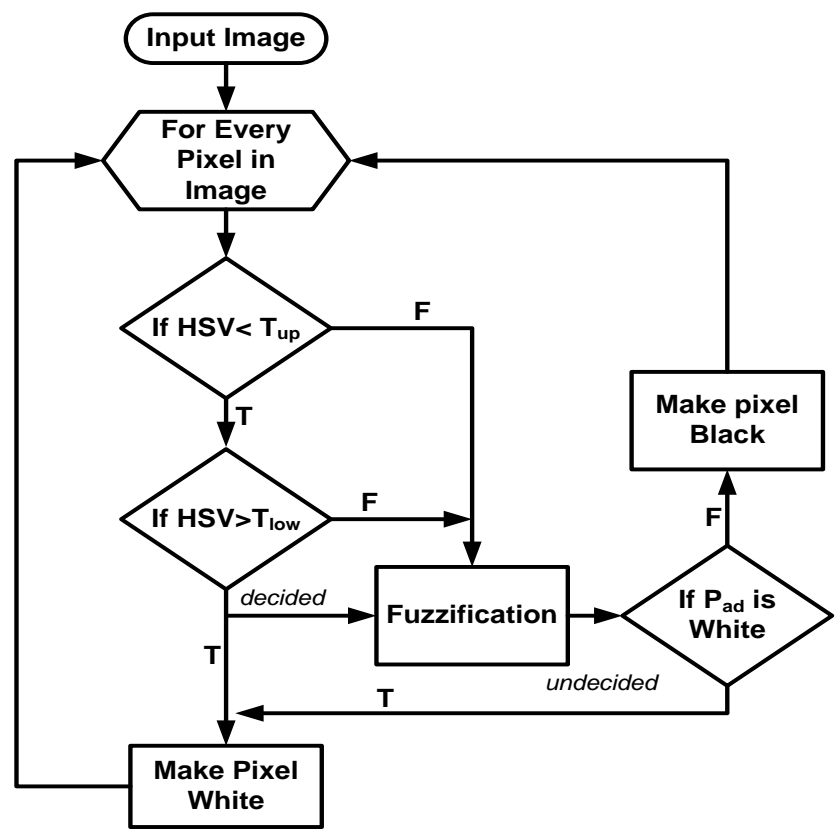

Fig. 2. Process flow of Proposed Image Binarization.

The first steps towards this implementation are to consider a sample of 100 images from the dataset (Fig. 3).

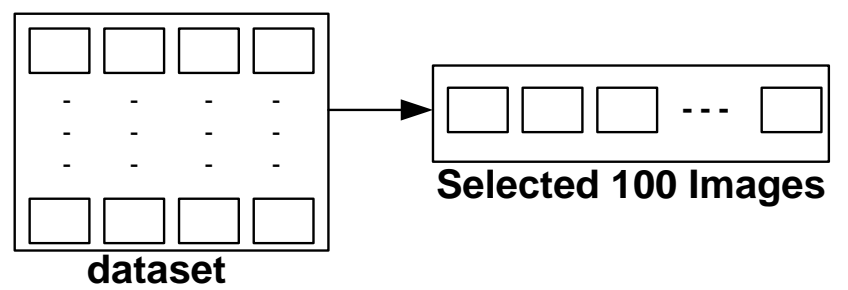

Fig. 3. Selection of Images from Dataset.

The next step of operation is to flatten every images and rearrange it into matrix $\mathrm{T}$.

Following are the mathematical operations being carried out:

$\mathrm{M}_{1}=$ Image $-1 \otimes \mathrm{I}$

$\mathrm{M}_{2}=$ Image $-2 \otimes \mathrm{I}$

$\mathrm{M}_{\mathrm{n}} 1=$ Image- $\mathrm{n} \otimes \mathrm{I}$

In the above expression (5), the variable $I$ represents identity matrix. Therefore, the resultant matrix $\mathrm{T}$ is mathematically expressed as follow,

$\mathrm{T}=\mathrm{M}_{1} \bowtie \mathrm{M}_{2} \bowtie \ldots \ldots \mathrm{M}_{\mathrm{n}}$ 
The next step of the operation will be to calculate and subtract mean as follows:

$T=\left[\begin{array}{cccc}v_{11} & v_{12} & \ldots & v_{1 m} \\ v_{21} & v_{22} & \ldots & v_{2 m} \\ \ldots & \ldots & \ldots & \ldots \\ v_{n 1} & v_{n 2} & \ldots & v_{n m}\end{array}\right]=A=\left[\begin{array}{c}\mu_{1} \\ \mu_{2} \\ \cdot \\ \mu_{n}\end{array}\right]$

$\mu_{i}=\frac{1}{m} \sum_{j=1}^{m} T_{i j}$

$\mathrm{m}=\mathrm{T}-\mathrm{A}$

Subtract every column of $\mathrm{S}$ with A. The next step of operation will be to compute the covariance matrix $\mathrm{S}$ as follows:

$S=\operatorname{Cov}(X)=\left[\begin{array}{llll}X_{1}\left(t_{1}\right) & X_{2}\left(t_{1}\right) & \ldots & X_{n}\left(t_{1}\right) \\ X_{1}\left(t_{n}\right) & X_{2}\left(t_{n}\right) & \ldots & X_{n}\left(t_{n}\right)\end{array}\right]$

The next process is to compute the eigenvalues for $\mathrm{S}$ matrix.

$\operatorname{det}(\mathrm{S}-\lambda \mathrm{I})=0$

Finally, an eigenvector is created

$E=\left[\begin{array}{c}\lambda_{1} \\ \lambda_{2} \\ \lambda_{n}\end{array}\right]$

Fig. 4 highlights the process involved in flattening the image.

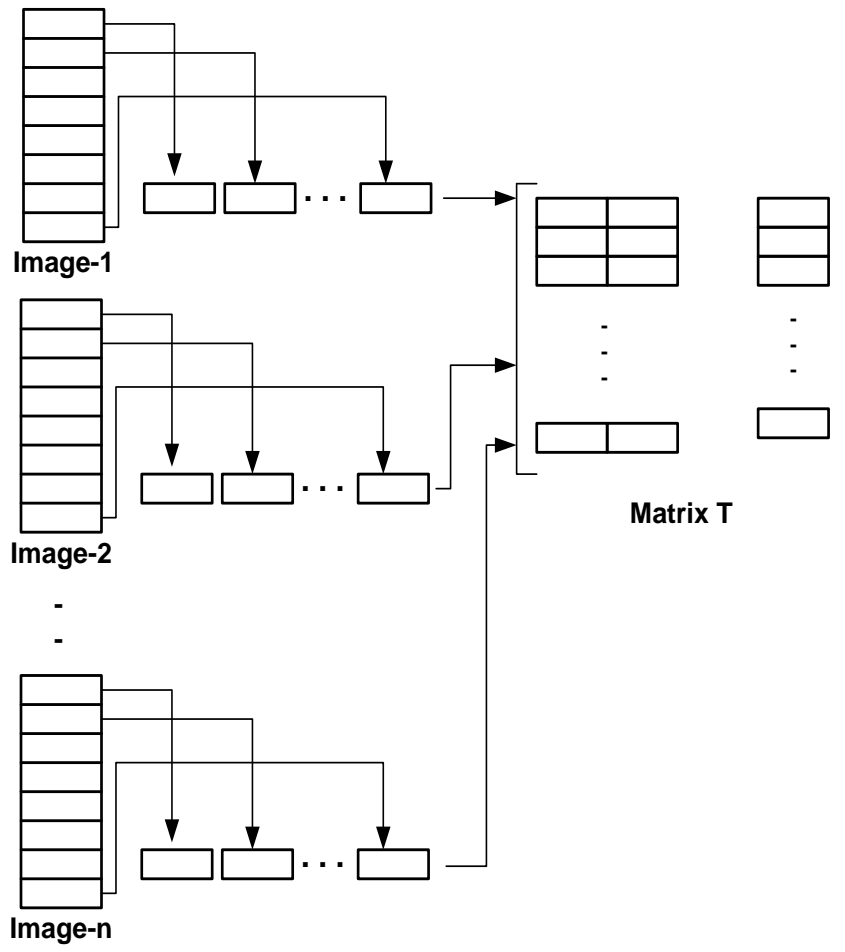

Fig. 4. Process of Flattening Image.
The step of fuzzification block is shown in Fig. 5 as follows.

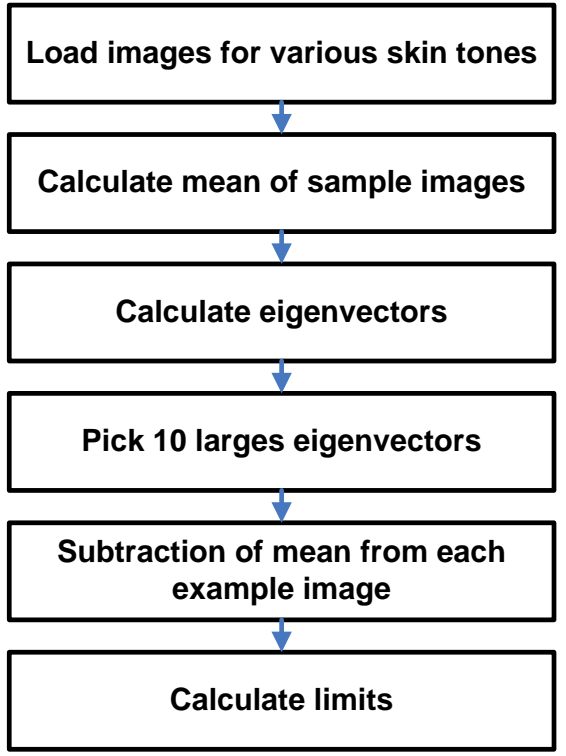

Fig. 5. Process Flow of Fuzzification.

\section{E. Image Enhancement}

This is the next steps of implementation after the binarization is carried out.In this process, the image is subjected for enhancement before passing it to the recognition algorithm. In this study hidden feature enhancements are being introduced which are not visible to human eyes however it will make a significant difference for the proposed system which will later contribute towards efficient authenticate a person. The final image will not be different from unprocessed images for human eyes.

\section{RESUlT AND DisCUSSION}

This section discusses about the results being obtained after implementing the proposed logic discussed in prior section. The proposed system consists of nine metadata which is tabulated below in Table I.

TABLE I. METADATA OF THE DATASET

\begin{tabular}{|l|l|l|}
\hline Sl. No. & Name & Description \\
\hline 1 & Id & Identity of the person to whom hand belongs to \\
\hline 2 & Age & Age of the person \\
\hline 3 & Gender & Gender of the person \\
\hline 4 & skinColor & Skin color of the person described by four types \\
\hline 5 & Accessories & If the person is wearing a ring \\
\hline 6 & nainPolish & If person has nail polish \\
\hline 7 & aspectOfHand & Side of hand and identifies left/right hand \\
\hline 8 & imageOfHand & Filename for image of hand \\
\hline 9 & Irregularities & If the person is handicap person \\
\hline
\end{tabular}


The proposed system is scripted in python over 64 bit machine. $11 \mathrm{~K}$ hands dataset has been chosen in this study since the dataset contain more images per person, higher resolution and other additional details [31][37]. The prime reason for selection of this dataset is because of its possession of maximum number of hand images for more than hundred number of subjects along with the presence of metadata. The contribution of the parameters used are: The parameter of minmax scaler $Y^{\prime}$ performs normalization, while $R^{\prime}, G^{\prime}, B^{\prime}$ is used for obtain pixel intensity information. From the algorithm of normalization, the effective control of RGB is carried out by parameters $\mathrm{C}_{\max }$ and $\mathrm{C}_{\min }$, while a logical condition is maintained in order to yield Hue $\mathrm{H}$ and Saturation Values S. Since it is observed that number of images per subject is not same for all, so there is a need to carry out analysis of the frequency of it in order to ensure that the data is balanced. The frequency of images is plotted per subject and the plot is exhibited in Fig. 5.

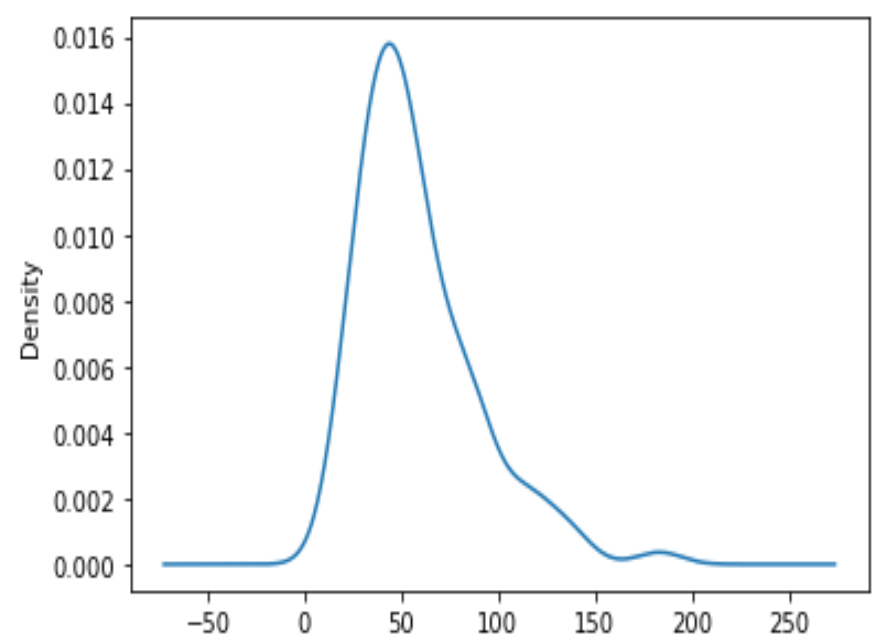

Fig. 6. Frequency Distribution of Images.

From Fig. 6, it can be observed that the data is not imbalanced as frequency doesn't deviate too much from mean/central tendency. As it can be observed, mean number of images per hand is 58.6. Minimum number of images per hand is 14 whereas maximum is 187 . The detailed numerical description of the same is shown in following Table II as follow:

TABLE II. STATISTICAL DESCRIPTION

\begin{tabular}{|l|l|}
\hline Items & Value \\
\hline Count & 189.00000 \\
\hline Mean & 58.603175 \\
\hline Standard Deviation & 30.841327 \\
\hline Minimum & 14.00000 \\
\hline Maximum & 187.00000 \\
\hline
\end{tabular}

The visual analysis of the proposed system is also carried out for the images corresponding to subjects with handicap situation as shown in Fig. 7. Analysis with this dataset gives that opportunity.

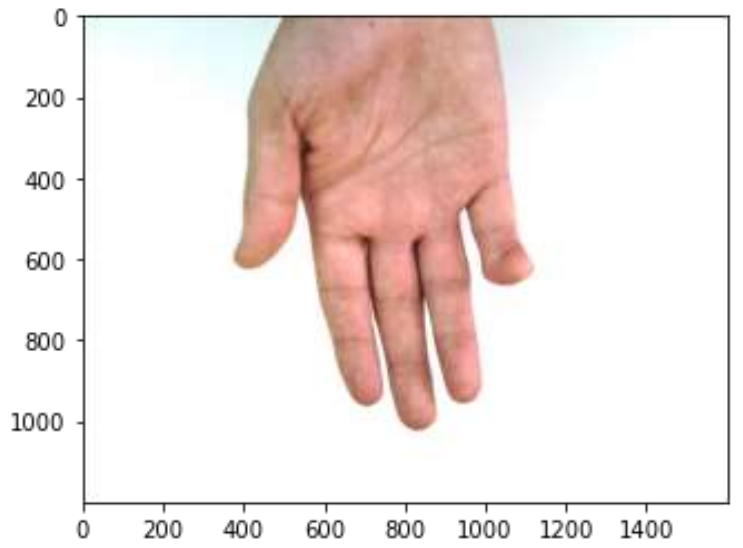

Fig. 7. Hand Image of Handicap Subject.

When the data if analyzed further, following conclusions can be drawn.

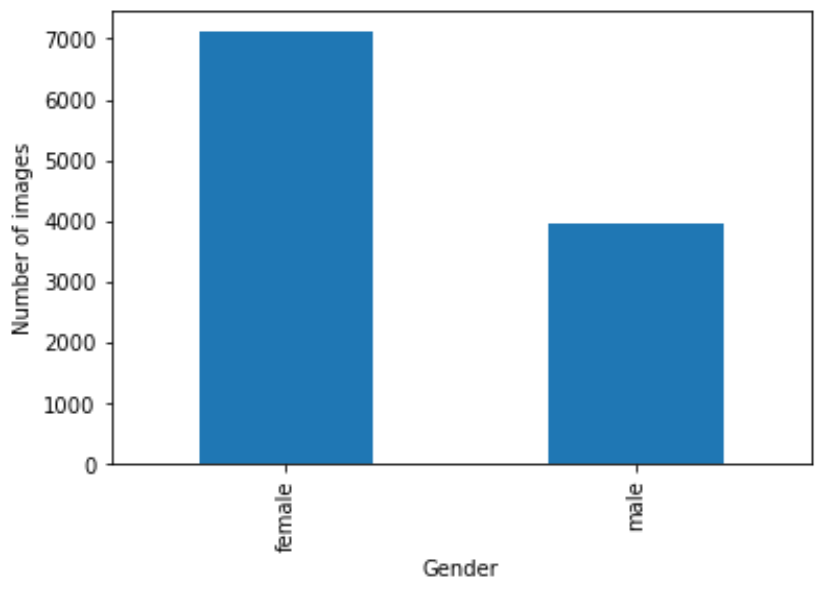

Fig. 8. Bar Graph of Female vs Male Hands.

There are around 7000 images of female hands whereas there are only around 4000 images of male hands as shown in Fig. 8. This will not pose many problems as there no significantly notable difference and gender recognition is not being perused in this study. Further, the proposed system also extracts information about Kernel Density Estimate (KDE) which is deployed for analyzing the probability density associated with the continuous variable.

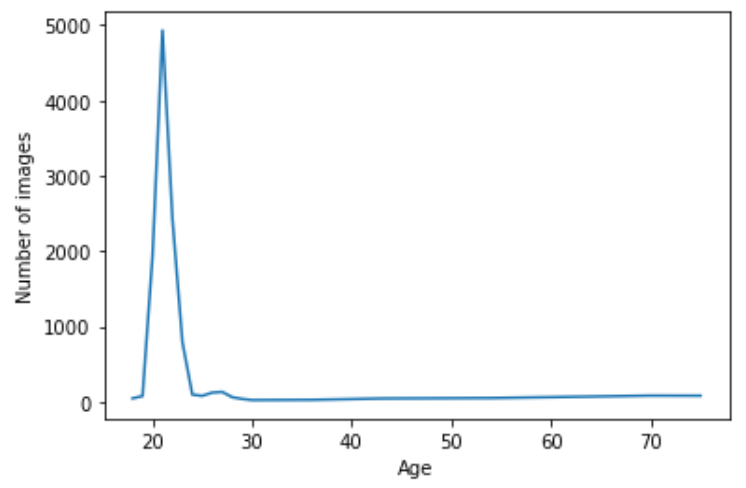

Fig. 9. KDE Plot for Age vs Number of Images. 
In above figure we can observe that most of the hand images are from people of age between 20 and 30 although, there are some images of the 70-year-old person as shown in Fig. 9.

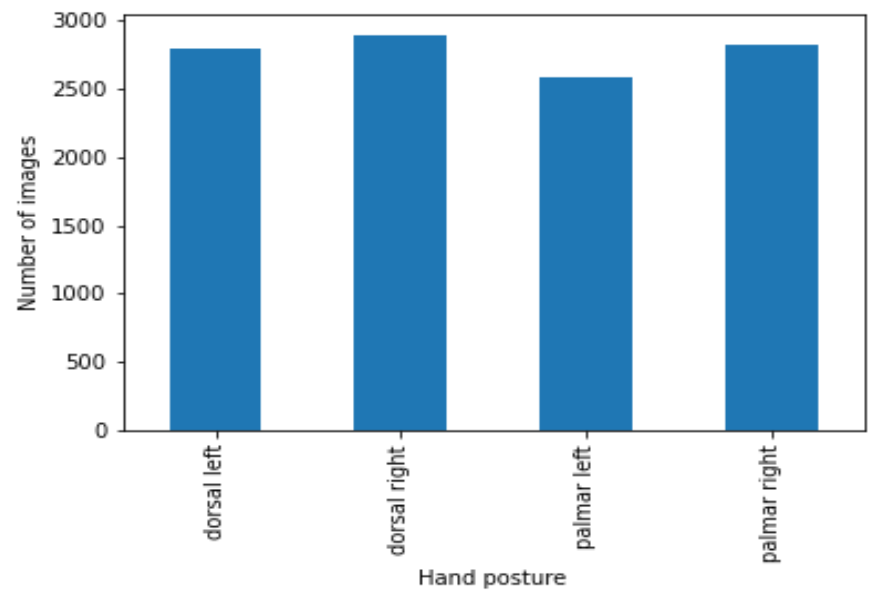

Fig. 10. Bar Graph of the Hand Poses.

As it can be observed above as shown Fig. 10, the images are evenly spread across among all four hand poses. Apart from this, it should be noted that image binarization is an important step in entire preprocessing algorithm. For every pixel within an image, the proposed system calculate whether it should be white in resulting image or black. Initially HSV values are considered. Based on all the images, considering all skin tones following minimum and maximum values are set by considering pixels belonging to skin of hand. Table III highlights the minimum and maximum values of HSV.

If any pixel value fall between these two maximum and minimum values then the pixel is made white by the algorithm. When the pixel is not within this range it could be possible that it is not within the range due to shadow and glaring effects. In order to avoid this phenomenon, proposed system uses fuzzy logic. In proposed fuzzification, the probability of the skin being part of actual skin is calculated by the help of eigenvectors. Eigenvectors are used to calculate a shape. They are nothing but set of vectors which represent the shape of the hand. The proposed system checks if the pixel falls within a shape of the hand in that particular image. In order to understand this system, the visual outcome exhibited in Fig. 6 represents the problems.

Fig. 11 shows the evident problems where middle finger and ringer finger are found to join with each other. This problem will eventually adversely affect the recognition process in later part. Hence, fuzzification significant contributes to address this problem. The fuzzy logic mechanism used in proposed system is very similar compared to the work of Chowdhury et al. [38] in which a very similar fuzzy logic algorithm is used to detect faces; however in this work, the equivalent process is being amended to detect hands. Any noises induced in this step will be removed by contour extraction as the algorithm considers only the larges contour among all. The next part of the visual analysis is that of image enhancement.
TABLE III. NUMERICAL OUTCOMES OF HSV

\begin{tabular}{|l|l|l|l|}
\hline Items & Hue & Saturation & Value \\
\hline Minimum & 20 & 10 & 60 \\
\hline Maximum & 40 & 165 & 255 \\
\hline
\end{tabular}

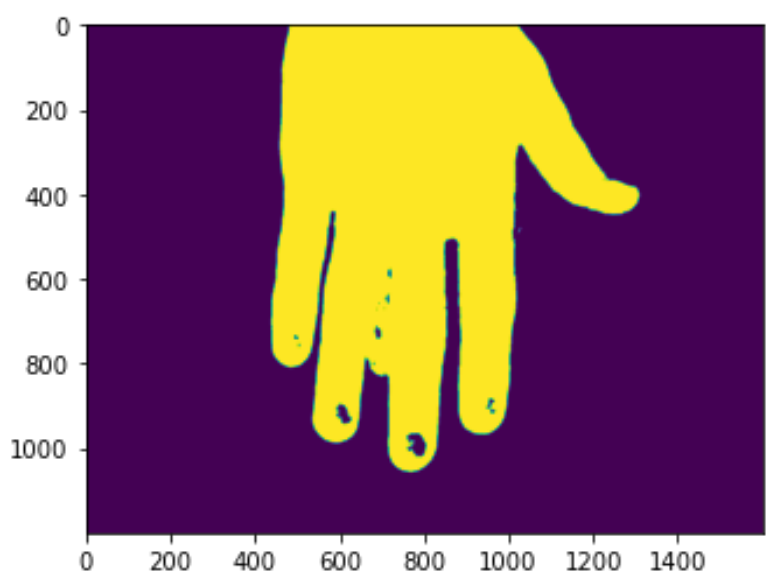

Fig. 11. Binarization without Fuzzy Logic.

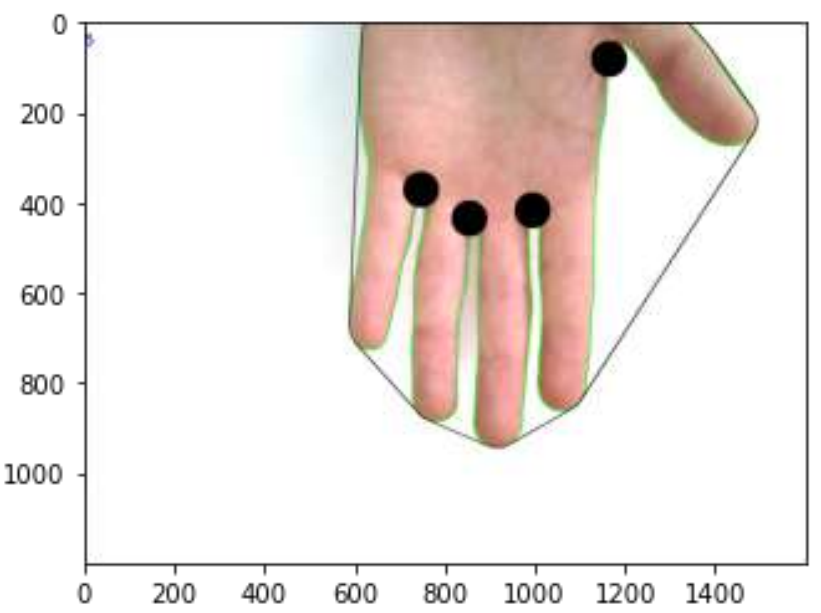

Fig. 12. Feature Enhancement Visible to Human Eyes.

Fig. 12 highlights the visual outcomes of enhancement operation

As it can be observed in above figure, majority the above features are enhanced. The contour is drawn around the hand, the out hull is drawn forming a polygon connecting the top most points of the hand, and finally the valleys in the contour are enhanced and these are the base of fingers. These are important features in hand geometry recognition.

The final stage of analysis is to carry out benchmarking of the proposed system by comparing with the existing system. For this purpose, the proposed system considers accuracy as performance parameters. Performance metrics of an authentication system is measured by Recognition accuracy. Formula for same has been provided below in expression (12).

Accuracy $=\frac{\text { Number of successful trials }}{\text { Total nuber of trials }} * 100$ 
Successful trial means if a person is authenticated the system should recognize him as authenticated or else it will recognize him as not authenticated. However, if it authorizes an unauthorized person and vice versa, it will be considered as failure case. Total number of trials is summation of the success cases and failure cases. Comparison is carried out with the existing work of Afifi et al. [31] who has presented two schemes side-by-side e.g. CNN with SVM and CNN integrated with Linear Binary Pattern (LBP) and SVM. The analysis of accuracy was carried out for three use cases of palm side with dark, medium, and fair skin tone as shown in Table IV.

TABLE IV. NuMERICAL OUTCOME OF PROPOSED STUdY

\begin{tabular}{|l|l|l|l|}
\hline Method & $\begin{array}{l}\text { Palmer side } \\
\text { - Dark skin }\end{array}$ & $\begin{array}{l}\text { Palmer side - } \\
\text { Medium skin }\end{array}$ & $\begin{array}{l}\text { Palmer side - } \\
\text { Fair skin }\end{array}$ \\
\hline CNN - SVM & 94.8 & 92.9 & 93.3 \\
\hline CNN - LBP - SVM & 96.0 & 95.3 & 95.6 \\
\hline Proposed method & 98.91 & 97.95 & 97.92 \\
\hline
\end{tabular}

The graphical outcome of the above mentioned tabulated data is showcased in the Fig. 13.

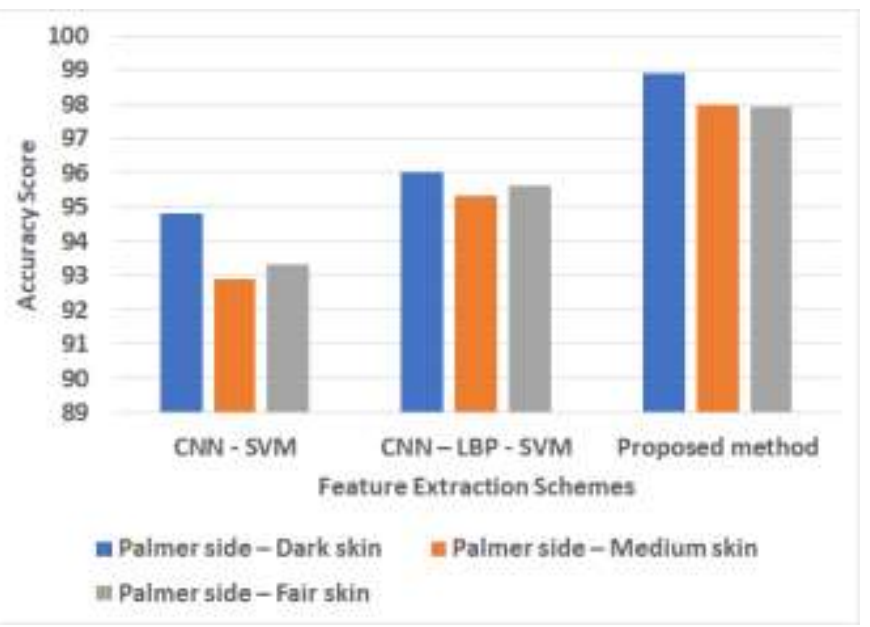

Fig. 13. Comparative Analysis Outcome.

The existing study of Afifi et al. [31] was able to produce $96 \%$ accuracy in most favorable conditions however the present work is able to show $98.91 \%$ accuracy which is a nearperfect system.

\section{CONCLUSION}

This paper has presented a discussion of a novel feature extraction mechanism where the input image is considered along with its meta-data. After subjecting to blurring, color space conversion, binarization, and image enhancement, the study outcome shows better accuracy achievement for proposed system. The experimental outcome of the proposed study shows that it has achieved comparatively higher accuracy in the range of $97.95-98.91 \%$ for three different forms of biometric images. This value of accuracy is significantly improved version when compared to existing approaches of feature extraction using machine learning approaches. Following are the contribution / novelty of proposed study viz.
- the proposed study presents a simplified approach unlike the complex and iterative approaches presented by existing literatures,

- the proposed scheme introduces a fuzzification mechanism in order to control the possible cases of outliers arising from challenging illumination condition between the fingers,

- without usage of training mechanism, unlike the evolving methods in existing literatures, the proposed system managed to offer higher accuracy that is numerically proven.

The limitation of the paper is that it doesn't offer any privilege for performing authentication using certain use-cases. It is because the development of the model is basically a computational framework which is meant to be integrated with any form of biometrics where the extracted features will be considered for authentication. It can be widely applied to any form of hand-based biometric system.

The future work of the proposed system can be extended towards developing an authentication system. The extracted features can be further trained and stored which can be used as a metadata while performing authentication with a query image.

\section{REFERENCES}

[1] Rui Z, Yan Z. A survey on biometric authentication: Toward secure and privacy-preserving identification. IEEE Access. 2018 Dec 27;7:59946009.

[2] "What is Biometrics Security", https://www.kaspersky.com/resourcecenter/definitions/biometrics, Retrived on 16-08-2021.

[3] K. Přihodová and M. Hub, "Biometric Privacy through Hand GeometryA Survey," 2019 International Conference on Information and Digital Technologies (IDT), 2019, pp. 395-401, doi: 10.1109/DT.2019.8813660.

[4] Sidlauskas DP, Tamer S. Hand geometry recognition. InHandbook of Biometrics 2008 (pp. 91-107). Springer, Boston, MA.

[5] C. Dhiman and D. K. Vishwakarma, "A Robust Framework for Abnormal Human Action Recognition Using \$boldsymbol $\{$ mathcal $\{\mathrm{R}\}\}$-Transform and Zernike Moments in Depth Videos," in IEEE Sensors Journal, vol. 19, no. 13, pp. 5195-5203, 1 July1, 2019, doi: 10.1109/JSEN.2019.2903645.

[6] Y. Lu, S. Yoon, S. Wu and D. S. Park, "Pyramid Histogram of Double Competitive Pattern for Finger Vein Recognition," in IEEE Access, vol. 6, pp. 56445-56456, 2018, doi: 10.1109/ACCESS.2018.2872493.

[7] P. Chen et al., "Design of Low-Cost Personal Identification System That Uses Combined Palm Vein and Palmprint Biometric Features," in IEEE Access, vol. 7, pp. 15922-15931, 2019, doi: 10.1109/ACCESS.2019.2894393.

[8] J. Lu, V. E. Liong and J. Zhou, "Simultaneous Local Binary Feature Learning and Encoding for Homogeneous and Heterogeneous Face Recognition," in IEEE Transactions on Pattern Analysis and Machine Intelligence, vol. 40, no. 8, pp. 1979-1993, 1 Aug. 2018, doi: 10.1109/TPAMI.2017.2737538.

[9] L. Fei, G. Lu, W. Jia, S. Teng and D. Zhang, "Feature Extraction Methods for Palmprint Recognition: A Survey and Evaluation," in IEEE Transactions on Systems, Man, and Cybernetics: Systems, vol. 49, no. 2, pp. 346-363, Feb. 2019, doi: 10.1109/TSMC.2018.2795609.

[10] L. Fei, B. Zhang, W. Jia, J. Wen and D. Zhang, "Feature Extraction for 3-D Palmprint Recognition: A Survey," in IEEE Transactions on Instrumentation and Measurement, vol. 69, no. 3, pp. 645-656, March 2020, doi: 10.1109/TIM.2020.2964076.

[11] B. Attallah, A. Serir, Youssef Chahir. Feature extraction in palmprint recognition using spiral of moment skewness and kurtosis algorithm. 
Pattern Analysis and Applications, Springer Verlag,2019, 22 (3), pp.1197-1205. 10.1007/s10044-018-0712-5.

[12] S. Bakheet and A. Al-Hamadi, "Robust hand gesture recognition using multiple shape-oriented visual cues", EURASIP Journal on Image and Video Processing, 2021.

[13] P. D. Deshpande, P. Mukherji and A. S. Tavildar, "Accuracy enhancement of biometric recognition using iterative weights optimization algorithm", EURASIP Journal on Information Security, 2019.

[14] Y. Zhang, Y. Li and J. Su, "Iterative learning control for image feature extraction with multiple-image blends", EURASIP Journal on Image and Video Processing, 2018.

[15] S. Yang, L. Gong and D. Qiao, "Image offset density distribution model and recognition of hand knuckle", EURASIP Journal on Image and Video Processing, 2019.

[16] F. Liu, S. Jiang, B. Kang and T. Hou, "A Recognition System for Partially Occluded Dorsal Hand Vein Using Improved Biometric Graph Matching," in IEEE Access, vol. 8, pp. 74525-74534, 2020, doi: 10.1109/ACCESS.2020.2988714.

[17] S. Zhao and B. Zhang, "Learning Salient and Discriminative Descriptor for Palmprint Feature Extraction and Identification," in IEEE Transactions on Neural Networks and Learning Systems, vol. 31, no. 12, pp. 5219-5230, Dec. 2020, doi: 10.1109/TNNLS.2020.2964799.

[18] P. Gupta and P. Gupta, "Multibiometric Authentication System Using Slap Fingerprints, Palm Dorsal Vein, and Hand Geometry," in IEEE Transactions on Industrial Electronics, vol. 65, no. 12, pp. 9777-9784, Dec. 2018, doi: 10.1109/TIE.2018.2823686.

[19] Z. Izakian, M. S. Mesgari, R. Weibel, "A feature extraction based trajectory segmentation approach based on multiple movement parameters", Elsevier-Engineering Applications of Artificial Intelligence, Vol.88, 2020.

[20] S. Ryu, J. Suh, S. Baek, S. Hong and J. Kim, "Feature-Based Hand Gesture Recognition Using an FMCW Radar and its Temporal Feature Analysis," in IEEE Sensors Journal, vol. 18, no. 18, pp. 7593-7602, 15 Sept.15, 2018, doi: 10.1109/JSEN.2018.2859815.

[21] M. Xin and Y. Wang, "Research on image classification model based on deep convolution neural network", EURASIP Journal on Image and Video Processing, 2019.

[22] C. Du, L. Zhang, X. Sun, J. Wang and J. Sheng, "Enhanced MultiChannel Feature Synthesis for Hand Gesture Recognition Based on CNN With a Channel and Spatial Attention Mechanism," in IEEE Access, vol. 8, pp. 144610-144620, 2020, doi: 10.1109/ACCESS.2020.3010063.

[23] M. K. Abd-Ellah, A. I. Awad, A. A. M. Khalaf, and H. F. A. Hamed, "Two-phase multi-model automatic brain tumour diagnosis system from magnetic resonance images using convolutional neural networks", EURASIP Journal on Image and Video Processing, 2018.

[24] G. Li, R. Zhang, M. Ritchie and H. Griffiths, "Sparsity-Driven MicroDoppler Feature Extraction for Dynamic Hand Gesture Recognition," in IEEE Transactions on Aerospace and Electronic Systems, vol. 54, no. 2, pp. 655-665, April 2018, doi: 10.1109/TAES.2017.2761229.
[25] M. J. J. Ghrabat, G. Ma, I. Y. Maolood, S. S. Alresheedi and Z. A. Abduljabbar, "An effective image retrieval based on optimized genetic algorithm utilized a novel SVM-based convolutional neural network classifier", Springer-Human-Centric Computing and Information Sciences, 2019.

[26] Z. Pan, J. Wang, Z. Shen, X. Chen and M. Li, "Multi-Layer Convolutional Features Concatenation With Semantic Feature Selector for Vein Recognition," in IEEE Access, vol. 7, pp. 90608-90619, 2019, doi: 10.1109/ACCESS.2019.2927230.

[27] C. Wu et al., "sEMG Measurement Position and Feature Optimization Strategy for Gesture Recognition Based on ANOVA and Neural Networks," in IEEE Access, vol. 8, pp. 56290-56299, 2020, doi: 10.1109/ACCESS.2020.2982405.

[28] C. Yoo, S. Ji, Y. Shin, S. Kim and S. Ko, "Fast and Accurate 3D Hand Pose Estimation via Recurrent Neural Network for Capturing Hand Articulations," in IEEE Access, vol. 8, pp. 114010-114019, 2020, doi: 10.1109/ACCESS.2020.3001637.

[29] G. Yuan, X. Liu, Q. Yan, S. Qiao, Z. Wang and L. Yuan, "Hand Gesture Recognition Using Deep Feature Fusion Network Based on Wearable Sensors," in IEEE Sensors Journal, vol. 21, no. 1, pp. 539-547, 1 Jan.1, 2021, doi: 10.1109/JSEN.2020.3014276.

[30] A. Wibisono and P. Mursanto, "Multi Region-Based Feature Connected Layer (RB-FCL) of deep learning models for bone age assessment", Springer-Journal of Big Data, 2020

[31] Mahmoud Afifi, "11K Hands: Gender recognition and biometric identification using a large dataset of hand images." Multimedia Tools and Applications, 2019.

[32] Pitaloka DA, Wulandari A, Basaruddin T, Liliana DY. Enhancing CNN with preprocessing stage in automatic emotion recognition. Procedia computer science. 2017 Jan 1;116:523-9.

[33] Ji H, Lu W, Shen L. Backbone Based Feature Enhancement for Object Detection. InProceedings of the Asian Conference on Computer Vision 2020.

[34] Kumar N, Nachamai M. Noise removal and filtering techniques used in medical images. Orient J. Comp. Sci and Technol. 2017 Mar;10(1).

[35] Oldal LG, Kovács A. Hand geometry and palmprint-based authentication using image processing. In2020 IEEE 18th International Symposium on Intelligent Systems and Informatics (SISY) 2020 Sep 17 (pp. 125-130). IEEE.

[36] Kolkur S, Kalbande D, Shimpi P, Bapat C, Jatakia J. Human skin detection using RGB, HSV and $\mathrm{YCbCr}$ color models. arXiv preprint arXiv:1708.02694. 2017 Aug 9.

[37] "11k Hands", https://sites.google.com/view/11khands, Retrived on 1608-2021.

[38] Chowdhury A, Tripathy SS. Human skin detection and face recognition using fuzzy logic and eigenface. In2014 International Conference on Green Computing Communication and Electrical Engineering (ICGCCEE) 2014 Mar 6 (pp. 1-4). IEEE. 